\title{
Radio-interferometric Neutrino Reconstruction for the Askaryan Radio Array
}

\author{
Ming-Yuan $\mathrm{Lu}^{1, \star}$ for the ARA Collaboration \\ ${ }^{1}$ Wisconsin IceCube Particle Astrophysics Center, University of Wisconsin,Madison, WI 53706, USA
}

\begin{abstract}
The Askaryan Radio Array (ARA) is a neutrino telescope array under phased deployment near the South Pole. The array aims to discover and determine the ultra-high energy neutrino flux via detection of the Askaryan signal from neutrino-induced showers. This novel detection channel makes ARA the most cost-effective neutrino observatory in probing the neutrino flux from $10^{17} \mathrm{eV}-10^{19} \mathrm{eV}$. This contribution will discuss an interferometric vertex reconstruction technique developed for ARA, taking into account the curved paths traveled by EM radiation in inhomogeneous ice. Preliminary results on the directional reconstruction of an in situ calibration pulser as well as simulated neutrino vertices will be presented.
\end{abstract}

\section{Introduction}

The existence of cosmic rays carrying energies up to over $10^{21} \mathrm{eV}$ poses a mystery in modern astrophysics [1]. No confirmed astrophysical objects accelerate cosmic rays to such energies. However, these ultra-high-energy cosmic rays (UHECRs) are expected to engage in photopion production with cosmic microwave background photons and give rise to UHE cosmogenic neutrinos [2-4]. This neutrino flux opens a window to profound physics such as cosmic ray composition, UHECR source evolution, and so on. Detection of such neutrinos should aid physicists in solving the UHECR puzzle.

We seek to search for these neutrinos via the Askaryan emission in radio frequency (RF) transparent ice. This technique allows for a cost-effective neutrino observatory sensitive to UHE neutrinos. First predicted by G.A. Askaryan [5], the Askaryan signal arises from coherent emission due to charge asymmetry built up in a neutrino-induced shower in dense media. The angular profile of this broadband radiation is centered at the Cherenkov angle, and the total power grows quadratically with neutrino energy. In ice, this radiation peaks from 0.1 to $1 \mathrm{GHz}$. Additionally, Antarctic ice near the South Pole has a measured RF attenuation length of $O(1 \mathrm{~km})$ [6], making it ideal for radio-Cherenkov based UHE neutrino experiments, such as the Askaryan Radio Array (ARA) [6]. ARA is designed to cover an area of $O\left(100 \mathrm{~km}^{2}\right)$, and is able to measure the timing, the frequency content, and the polarization of the said Askaryan signal. Such rich information, in principle, grants us access to neutrino vertex position, emission angle, and travel direction. Here we describe an approach to reconstruct the vertex position.

^e-mail: mlu27@icecube.wisc.edu 


\section{Experimental setup}

An ARA station hosts four measurement strings and two calibration strings down to $200 \mathrm{~m}$ depth. Each measurement string has four broadband antennas. Two are vertically polarized (Vpols), and the other two are horizontally polarized (Hpols). The calibration strings sit roughly 40m away from the station center (geometric center of all 16 measurement antennas). One selected calibration pulser antenna (calpulser) transmits at 1 PPS to a station at a time with a fixed polarization. We run a simple multiplicity trigger, where 3 power excursions out of $8 \mathrm{Vpol}$ or Hpol channels within a pre-set duration form a trigger. Once a trigger occurs, digitization at $3.2 \mathrm{GS} / \mathrm{s}$ yields a waveform of several hundred nanoseconds for each channel. A collection of such waveforms is an event. For details, see [6, 7].

\section{Reconstruction method}

Relative differences in signal arrival times between antennas are needed to calculate the cross correlation (CC) values for interferometry. The Antarctic ice has a depth-dependent index of refraction. This causes the rays of electromagnetic waves traversing the ice to bend downward. A raytracing code named RaySolver in the ARA simulation package AraSim [8] implements a semi-analytic algorithm to derive these rays. A significant consequence of the ray-bending effect is that some region of ice becomes invisible to a receiver. This region is called the firn shadow. Note also that for a source outside the firn shadow, more than one path to the receiver is possible. These phenomena are showcased in Fig. (1). In the next section, we present Radiospline, a software library for fast and precise signal arrival time determination taking into account ray-bending effects.
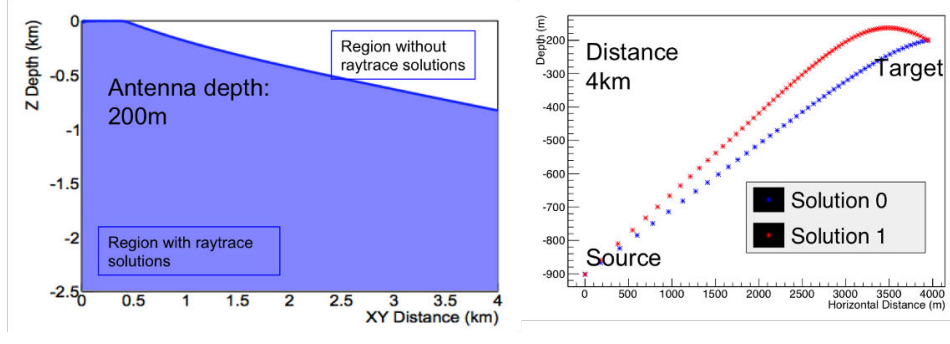

Figure 1. Left: Region where a ray path can connect from source to target is shaded. Firn shadow is blank [8]. Right: Ray paths calculated by RaySolver. The horizontal distance between the source and target is $4 \mathrm{~km}$. Solution 0 marks the direct ray and Solution 1 marks the indirect ray.

\subsection{Radiospline and interferometry}

Radiospline interpolates signal arrival times from a table of RaySolver outputs. Assuming azimuthal symmetry of ice, choosing cylindrical coordinates reduces the table to 3 dimensions: source depth, target depth, and the horizontal separation between the two. The source position moves vertically between $[-2800 \mathrm{~m}, 100 \mathrm{~m}]$ and horizontally between $[0 \mathrm{~m}, 5000 \mathrm{~m}]$, while the target depth varies within [-250m, $-20 \mathrm{~m}]$, all in steps of $10 \mathrm{~m}$. The table records arrival times from all geometric combinations. Basis spline fits are used to interpolate these time values. Basis splines are piece-wise polynomials that can be placed in linear combinations to form a fitting curve on a multi-dimensional set of data points. In practice, for a putative vertex location, Radiospline first determines if the vertex is in the firn shadow. If so, no arrival time is computed. If otherwise, source-in-ice or source-in-air tables are interpolated to give arrival times. The precision of the interpolation is $O(100 \mathrm{ps})$, while the computation time compared to direct use of RaySolver is reduced by a factor of $>500$. Note that for a source-target pair, only the direct ray is tabulated in Radiospline in this work. 


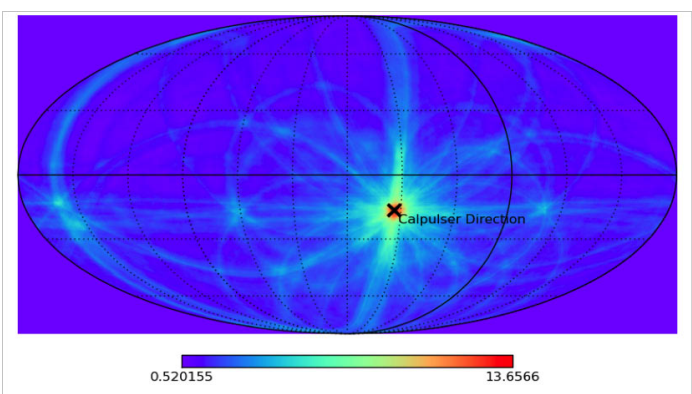

Figure 2. Reconstruction skymap of 2013 ARA3 run \#673 cal event \#2. Color represents the coherence value [arb. unit]. The black cross marks the known direction of the calpulser in operation at the time of event recording.

To perform interferometry, we start from a Healpix [9] skymap assumed to be surrounding our station center at a fixed radial distance. We treat each pixel center as a putative vertex position. Thus, each pixel gives a set of signal arrival times for the antennas from Radiospline. These arrival times are converted to relative time delays between channels. We then construct $\mathrm{CC}$ functions from the waveforms of every pair of same-polarization channels. According to the delays of each pixel, CC values are obtained and projected back to the skymap. We define the "coherence" to be the sum of all CCs projected on the same pixel, and build a skymap where each pixel holds a coherence value as proxy to the aligned power. The pixel with the maximal coherence gives the reconstructed direction. All skymaps presented here have 196,608 pixels. Mean pixel spacing is $0.4581^{\circ}$. One can repeat this process for a set of skymaps at distinct radial distances from the station center and attempt full vertex position reconstruction including the vertex distance. For this, 50 skymaps are generated at radial distances from $100 \mathrm{~m}$ to $5000 \mathrm{~m}$ in $100 \mathrm{~m}$ steps. Note that all following reconstruction uses only Vpol channels.

\section{Application to Data}

\subsection{Calpulser reconstruction}

Here we fix the skymap radial distance to $42 \mathrm{~m}$, the known calpulser distance. In general we are able to reconstruct the calpulser direction to $\sim<1^{\circ}$ accuracy. An example event is shown in Fig. (2). The coherence peak is prominent and aligns with the true direction marked by a black cross. Table 1 lists the mean reconstructed zenith and azimuth for a month of filtered calpulser events versus the true values. We conclude that the reconstruction of the Vpol calpulser is successful.

Table 1. Comparison of the reconstructed zenith and azimuth means to the true values of the ARA 3 calpulser.

\begin{tabular}{llll}
\hline & True & Reco Mean & Difference \\
\hline Zenith $\left[^{\circ}\right]$ & 106.3 & 106.2 & -0.1 \\
Azimuth $\left[{ }^{\circ}\right]$ & 63.8 & 63.5 & -0.3 \\
\hline
\end{tabular}

\subsection{Simulation reconstruction}

The simulated data are $10^{18} \mathrm{eV}$ neutrinos, where indirect rays are excluded. An example event is shown in Fig. (3). The skymap exhibits a clear hotspot that indicates well the vertex direction. The reconstructed [zenith, azimuth] is $\left[102.94^{\circ}, 222.89^{\circ}\right.$ ], with the true direction $\left[102.74^{\circ}, 222.69^{\circ}\right.$ ].

A set of skymaps at different radial distances can also be constructed as described earlier. 2658 triggered AraSim events are reconstructed this way. We apply a quality cut on the maximal coherence 

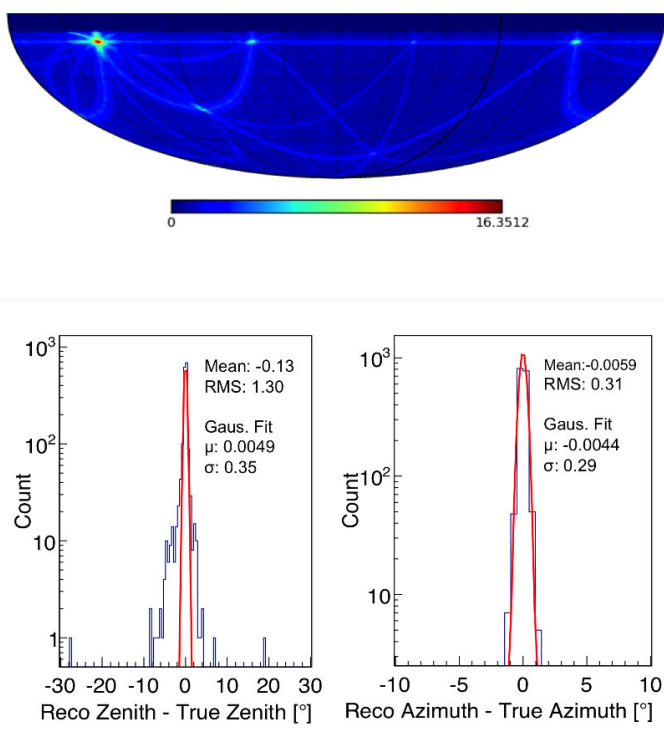

Figure 3. A $10^{18} \mathrm{eV}$ simulated neutrino event reconstructed on the lower half of the skymap. Simulation-true vertex distance of $2581 \mathrm{~m}$ was assumed in the construction of the skymap. The hotspot gives the reconstructed vertex direction.

Figure 4. $10^{18} \mathrm{eV}$ simulated neutrino events vertex direction reconstruction. Plots show the difference of reconstructed zenith/azimuth from true values after maximal coherence value $>5.0$ cut. The fitting curves represent Gaussian fits to the histograms.

and demonstrate that in principle a zenith resolution of $1.3^{\circ}$ and a azimuth resolution of $0.31^{\circ}$ can be achieved (Fig. (4)). The cut efficiency is being studied. We should also mention that this reconstruction fails to resolve the true vertex distance. This, combined with ray-bending, constrains our ability to reach sub-degree zenith angle resolution. However, preliminary studies with expanded antenna spacing show success in vertex distance reconstruction up to several hundred meters.

\section{Summary and future plan}

An interferometric reconstruction for ARA using the Radiospline library is developed. We demonstrate the ability to reconstruct the calpulser direction with ARA3 data, and we are able to reconstruct the direction of simulated events to $\sim 1^{\circ}$ angular resolution under stated quality cuts. If implemented on a parallel-computing framework of GPUs, the interferometric techniques can potentially be used as online filters for background rejection. Moving forward, we plan to finalize the cuts and fully unblind at least two years (2013-14) of ARA2 and ARA3 data for a diffuse neutrino search. Possible ways to improve the reconstruction include updating Radiospline to describe indirect rays and applying more sophisticated methods, such as machine learning algorithms, to extract information from skymaps.

\section{References}

[1] J. Abraham et al. (Auger Collaboration), Phys. Lett. B 685, 239 (2010)

[2] K. Greisen, Phys. Rev. Lett. 16, 748 (1966)

[3] G. T. Zatsepin and V. A. Kuzmin, JETP Lett. 4, 78 (1966)

[4] V. S. Beresinsky, G. T. Zatsepin, Phys. Lett. B 28, 423 (1969)

[5] G. A. Askaryan, JETP 14, 441 (1962)

[6] P. Allison et al. (ARA), Astropart. Phys. 35, 457, (2012)

[7] P. Allison et al. (ARA), Phys. Rev. D 93, 082003 (2016)

[8] P. Allison et al. (ARA), Astropart. Phys. 70, 62 (2015)

[9] Gorski, K. M. et al., ApJ 622, 759 (2005) 